\title{
The efficacy of long-lasting nets with declining physical integrity may be compromised in areas with high levels of pyrethroid resistance
}

\author{
Eric O Ochomo ${ }^{1,2^{*}}$, Nabie M Bayoh ${ }^{1}$, Edward D Walker ${ }^{3}$, Bernard O Abongo ${ }^{1}$, Maurice O Ombok ${ }^{1}$, Collins Ouma ${ }^{2}$,
} Andrew K Githeko ${ }^{4}$, John Vulule ${ }^{4}$, Guiyun Yan ${ }^{5}$ and John E Gimnig ${ }^{6}$

\begin{abstract}
Background: Long-lasting insecticide-treated mosquito nets (LLINs) are a primary malaria prevention strategy in sub-Saharan Africa. However, emergence of insecticide resistance threatens the effectiveness of LLINs.

Methods: Cross-sectional surveys of LLINs were conducted in houses of seven and four villages in Gem and Bungoma Districts in western Kenya, respectively. Condition (number and area of holes in the nets), number and species of mosquitoes resting inside them, and insecticidal activity of nets were quantified. Mosquitoes collected inside nets were allowed to lay eggs and progeny tested for susceptibility to deltamethrin and permethrin, pyrethoids commonly deployed in LLINs in western Kenya.
\end{abstract}

Results: In Gem, $83.3 \%$ of nets were less than three years old and $32.4 \%$ had at least one hole of any size; while in Bungoma, 92\% were less than three years old and $48 \%$ had at least one hole. No anopheline and five Culex spp. mosquitoes were found resting inside nets in Gem regardless of the number and size of holes, while 552 Anopheles gambiae s.l., five Anopheles funestus s.l. and 137 Culex spp. were in nets in Bungoma. The number of mosquitoes resting inside nets increased with hole areas $>50 \mathrm{~cm}$ in Bungoma. In WHO resistance assays, f1 offspring of samples collected in nets in Bungoma were 94 and 65\% resistant to deltamethrin and permethrin, respectively. Nets from Bungoma retained strong activity against a susceptible laboratory strain, but not against $\mathrm{f1}$ offspring of fieldcollected An. gambiae s.s. All An. gambiae s.s. samples collected in nets were homozygous for the kdr genotype L1014S.

Conclusions: In areas with pyrethroid resistant vectors, LLINs with modest hole areas permit mosquito entry and feeding, providing little protection against the vectors. LLIN formulations develop large holes within three years of use, diminishing their presupposed lifetime effectiveness.

\section{Background}

Insecticide-treated nets (ITNs) are an important tool to protect individuals against the morbidity and mortality caused by malaria [1-3]. The distribution of long-lasting insecticidal nets (LLINs) (factory-treated ITNs designed to retain insecticidal activity for up to three years [4]) by governments, non-governmental organizations (NGOs) and donors, has resulted in a dramatic increase in their

\footnotetext{
* Correspondence: ericochomo@yahoo.com

'KEMRI/CDC Research and Public Health Collaboration, PO Box 1578, Kisumu 40100, Kenya

${ }^{2}$ Department of Biomedical Science and Technology, School of Public Health and Community Development, Maseno University, Maseno, Kenya Full list of author information is available at the end of the article
}

ownership and contributed to the decline in malaria burden since 2000 [5]. Ownership and use of ITNs within households, as measured by the number of children under five years of age reported to have used an ITN the previous night, increased by three to tenfold between 2000 and 2008 in many African countries [5-7]. According to the latest World Malaria Report, $41 \%$ of children and $33 \%$ of all persons residing in malaria-endemic regions of sub-Saharan Africa reported sleeping under an ITN [5]. The distribution of LLINs in malaria endemic areas of Kenya has increased household ownership of any net to $70 \%$ and household ownership of an ITN to $60 \%$ [8]. The increase in ownership and use of ITNs has contributed to a significant decline in the burden of 
malaria throughout sub-Saharan Africa [5], including Kenya $[9,10]$.

All mosquito nets act as a physical barrier, preventing access to vector mosquitoes and thus providing personal protection against malaria to the individual(s) using the nets [11]. However, untreated nets with even a few holes provide little protection [12]. The addition of pyrethroid insecticides serves to enhance the effectiveness of intact mosquito nets and to extend the effectiveness of nets with holes. The pyrethroids used to treat the ITNs have an exito-repellent effect, thus adding a chemical barrier to the physical one and enhancing personal protection by nets $[11,13]$. The insecticides incorporated in the ITNs kill malaria vectors that come into contact with them and when used by a majority of the target population, may provide protection for the entire community, including those who do not themselves sleep under an ITN [14-16]. A meta-analysis of data from trials of treated and untreated nets suggested that approximately half of the protection was derived from the physical barrier of the net and half from the chemical barrier [17].

In western Kenya, malaria transmission in the lowland areas around Lake Victoria has historically been very high, with entomological inoculation rates estimated to be as high as $300+$ infectious bites per person per year [18-20]. However, the scaling up of ITNs and other malaria control tools has reduced transmission and malariaassociated morbidity and mortality. Between 2003 and 2007 , demographic surveillance indicated a $42 \%$ reduction in all-cause mortality among children less than five years of age coinciding with a scale up of ITNs as well as improvement in diagnostics and introduction of ACT [21]. Anopheles gambiae s.l. and Anopheles funestus densities declined markedly in a randomized trial of permethrintreated bed nets in a comparison of treatment versus control villages in western Kenya [22,23]. Subsequent monitoring demonstrated a decline in the proportion of $A n$. gambiae s.s., the principal vector of malaria, relative to Anopheles arabiensis [24].

However, a resurgence in malaria vectors, parasite prevalence and malarial disease burden has been observed in several sites in western Kenya despite high ownership of ITNs $[25,26]$. This resurgence could be due to one or a combination of the following factors: reduced efficacy of ITNs, insecticide resistance in mosquitoes, improper use of ITNs, stock-outs of anti-malarial drugs or even a poor dosing regimen of policy recommended drugs by private outlets [25,27]. This study investigated the impact of high levels of pyrethroid resistance in An. gambiae on their tendency to enter and rest inside nets. The results suggest that pyrethroid resistance in mosquitoes may undermine the effectiveness of nets with even a few holes. These findings have serious implications for malaria control programmes in
sub-Saharan Africa where resistance to pyrethroid insecticides is increasing rapidly [28].

\section{Methods}

\section{Study sites}

Bed net surveys were conducted in seven villages in Gem district $\left(0^{\circ} 07^{\prime} 30.06^{\prime \prime} \mathrm{N}, 34^{\circ} 24^{\prime} 32.56^{\prime \prime} \mathrm{E}\right)$ in Siaya County and four villages in Bungoma district $\left(0^{\circ} 35^{\prime}\right.$ $20.85^{\prime \prime} \mathrm{N}, 34^{\circ} 29^{\prime} 13.23^{\prime \prime}$ E) in the month of May 2013. The two study sites are about $100 \mathrm{~km}$ apart. Inhabitants of Bungoma include subsistence and large-scale farmers growing cash crops such as sugar cane, tobacco, onions, and tomatoes. Residents of Gem are primarily subsistence farmers growing food crops. The ITN coverage in the two sites is 80 and $70 \%$ for Gem and Bungoma, respectively $[10,29]$.

Malaria transmission in both areas is high. The main malaria vectors are An. gambiae s.s., An. arabiensis and Anopheles funestus. Previous studies have shown high resistance to pyrethroids [30] in An. gambiae in both areas. The species composition of these areas differs. Anopheles gambiae comprises $>70 \%$ of the An. gambiae s.l. mosquitoes collected from houses in Bungoma, whereas An. arabiensis accounted for up to $90 \%$ of the An. gambiae s.l. in Gem and surrounding areas [24].

\section{Bed net cross-sectional survey}

Community-based, cross-sectional surveys were carried out in the two study sites. Sampling was done in all the houses in the selected villages in Bungoma and in randomly selected houses in the selected villages in Gem. The surveys consisted of interviews with household heads and an inspection of all nets in the house. Interviews were conducted using a structured questionnaire administered on personal data assistants (PDAs, Dell Axim X50, Dell Inc, Dallas, TX, USA) and collected information on the household characteristics: wall type, roof type, whether eaves were open or closed, insecticide use and application within the household, frequency of net use, the number of people who used the nets the previous night and the brands and ages of the nets. The interviewers examined the nets for the presence of mosquitoes resting inside them using torches. Any mosquitoes observed were collected using a mouth aspirator, transferred into a paper cup and labeled with the house ID and net type. The samples were placed in a cool box and maintained on a $10 \%$ sugar solution for transport to insectary. The interviewers then examined each net and recorded the presence, number and size of holes. Hole sizes were categorized using methods recommended by the WHO Pesticide Evaluation Scheme: the thumb was used to estimate hole sizes $\leq 2 \mathrm{~cm}$ in diameter (small), holes larger than the thumb but smaller than the fist were estimated to be between 2 and $10 \mathrm{~cm}$ (medium), while 
those larger than the fist were estimated to be $\geq 10 \mathrm{~cm}$ (large). Holes that fell within the largest hole size category $(>25 \mathrm{~cm}$ ) recommended by WHOPES [31] were recorded as large holes $(\geq 10 \mathrm{~cm})$.

\section{Mosquito rearing}

Female mosquitoes were sorted based on species ( $A n$. gambiae s.l. and An. funestus s.l.) and on abdominal status. Fed and half-gravid samples collected from the nets were maintained on 10\% sugar solution at the KEMRI/ CDC insectary until they became gravid. Gravid mosquitoes were placed in oviposition cups containing laying pads made of moist cotton wool covered with filter paper. Mosquitoes were pooled into 34 oviposition cups with one to five females per cup. To ensure maximum hatch rate, once the females laid eggs, the laying pads with eggs were transferred to a hatching bowl. Hatched larvae from each cup were transferred to a larval tray. Larvae were fed daily on a mixture of brewer's yeast and fish food, and their water changed every two days. Pupae from the same larval tray were transferred to the same eclosion cup and placed inside individual paper cups for emergence. Freshly emerged adults were fed on 5\% sugar solution for three days after which they were ready for bioassays. In addition, mosquitoes were collected as larvae from each site and reared until three days old adults as above.

\section{Susceptibility testing}

To assess susceptibility to insecticides, field collected mosquitoes (three days old) were exposed to permethrin and deltamethrin using WHO tube tests [32]. Three sets of samples were exposed: f1 offspring of the Anopheles samples collected inside mosquito nets from Bungoma and adult samples from larval collections in Bungoma and Gem.

\section{Bed net bioassays}

In Bungoma, 68 of the nets with mosquitoes resting inside and 31 nets without were collected from the field and the owners provided with new LLINs in exchange. WHO cone bioassays using five to ten mosquitoes on five pieces cut from these nets, one piece per side and one from the top [33] were performed using the susceptible $A n$. gambiae s.s. Kisumu strain. In addition, the progeny of females collected from inside nets were exposed to new, unused, unwashed PermaNet 2.0 (Vestergaard Frandsen SA, Aarhus, Denmark) and Olyset (Sumitomo Chemicals, Osaka, Japan) as these were the primary net brands observed in the field. For all bioassays, mosquitoes were exposed in plastic cones for $3 \mathrm{~min}$ and then transferred to holding cages with access to $10 \%$ sucrose solution. Knockdown was recorded
60 min after exposure and mortality was recorded 24 hours after exposure.

\section{Molecular assays}

Conventional polymerase chain reaction (PCR) was used to distinguish between the two sibling species of the $A n$. gambiae species complex native to western Kenya, $A n$. gambiae s.s. and An. arabiensis [34]. Further, the mosquito samples were tested for the presence of the $1014 \mathrm{~S}$ $k d r$ genotype using the methods previously described [35] and as modified in Mathias et al. [36]. Sporozoite ELISA was also conducted on the head and thorax of all the female mosquitoes [37].

\section{Data analysis}

The average hole area was estimated according to the methods recommended by the WHO Pesticide Evaluation Scheme [33]. The data are presented in square centimetres instead of as a proportionate hole index. A Poisson regression model, corrected for over-dispersion, was used to estimate the effect of net type, net age and the physical condition of the net, and the number of mosquitoes resting inside. The hole area was categorized as $\leq 50 \mathrm{~cm}^{2}, 50-500 \mathrm{~cm}^{2}$ and $>500 \mathrm{~cm}^{2}$. Nets with no holes were used as the reference category in the regression analysis.

\section{Ethical clearance}

Ethical clearance was obtained from the Ethical Review Committee of the Kenya Medical Research Institute (SSC 2267) and the Institutional Review Boards of the US Centers for Disease Control and Prevention (IRB 6395).

\section{Results}

\section{Bed net cross-sectional survey}

The bed net survey was conducted in a total of 482 households: 303 in Bungoma and 179 in Gem, with a total of 590 nets being sampled. The numbers of the different types of nets and their ages are shown in Table 1. In Gem, just over half $(117 / 216,54.0 \%)$ of the nets were PermaNets while 69/216 (32.1\%) were Olyset nets. In Bungoma, most of the nets were Olyset nets $(279 / 374$, $74.7 \%)$ while only 58/374 (15.6\%) were PermaNets. The remainder of the nets sampled in both sites (57/590, 6.7\%) were SupaNets [commercially available, conventionally treated nets bundled with deltamethrin treatment kits $\left.\left(\mathrm{KO}-\mathrm{Tab}^{\circ}\right)\right]$ or nets that could not be identified. In both sites, most nets were less than three years old; $84.5 \%$ were less than or equal to three years old in Gem and $91.6 \%$ were less than or equal to three years old in Bungoma. The percentage of nets with at least one hole was $32.4 \%$ in Gem and $48 \%$ in Bungoma. Five Culex spp. mosquitoes and no anophelines were 
Table 1 Number of each type of bed net in the two study sites

\begin{tabular}{|c|c|c|c|c|c|c|}
\hline \multirow[t]{2}{*}{ Type of ITN } & \multicolumn{3}{|c|}{ Gem } & \multicolumn{3}{|c|}{ Bungoma } \\
\hline & $\leq 3$ years & $>3$ years & Unknown & $\leq 3$ years & $>3$ years & Unknown \\
\hline Olyset & 59 (85.5) & $10(14.5)$ & $0(0.0)$ & $254(91.7)$ & $14(5.1)$ & $9(3.3)$ \\
\hline Permanet 2.0 & $96(82.1)$ & $20(17.1)$ & $1(0.9)$ & 48 (78.7) & $8(13.1)$ & $5(8.2)$ \\
\hline Supanet* & $1(33.3)$ & $2(66.7)$ & $0(0.0)$ & $27(75.0)$ & $8(22.2)$ & $1(2.8)$ \\
\hline Other & 24 (88.9) & $3(11.1)$ & $0(0.0)$ & 0 & 0 & 0 \\
\hline Total & $180(83.3)$ & 35 (16.2) & $1(0.5)$ & $329(88.0)$ & $30(8.0)$ & $15(4.0)$ \\
\hline
\end{tabular}

Numbers in parentheses indicate the percent of each net type that are less or equal to 3 or than or greater than three years of age as reported by the owner in each site.

*A conventional net available at local shops.

collected from inside nets in Gem while in Bungoma, a total of 552 An. gambiae s.l., five An. funestus s.l. and 137 Culex spp. were collected inside nets. Hereafter, results are reported only for Bungoma.

Overall, 36\% of nets in Bungoma had at least one Anopheles mosquito resting inside the net. Among the different net types, $38 \%$ of the Olyset nets, $33 \%$ of the Permanet 2.0 nets and 29\% of all Supanet nets had at least one An. gambiae s.l. resting inside. The proportion of nets less than or equal to three years old and those older than three years that had at least one mosquito resting inside were 36.9 and $36.6 \%$, respectively. The proportion of intact nets (without holes) and damaged nets (with one or more holes) that had at least one mosquito resting inside were 37 and $48 \%$, respectively.

The mean number of Anopheles mosquitoes found within nets increased in nets with hole sizes above $50 \mathrm{~cm}^{2}$ (Table 2). The number of mosquitoes collected from nets with total hole areas less than $50 \mathrm{~cm}^{2}$ was not significantly different from nets with no holes. For nets with hole areas $50-500 \mathrm{~cm}^{2}$ and $>500 \mathrm{~cm}^{2}$, there were generally twice as many mosquitoes observed resting inside compared to nets with no holes $(\mathrm{RR}=2.08, P=0.001$ for $50-500 \mathrm{~cm}^{2} ; \mathrm{RR}=1.89, \quad P=0.012$ for $>500 \mathrm{~cm}^{2}$ ) (Table 3). There was no increase in the number of mosquitoes found resting in nets with hole areas $>500 \mathrm{~cm}^{2}$ compared to nets with hole areas between $50-500 \mathrm{~cm}^{2}$ suggesting a threshold effect. There was no significant difference in the number of mosquitoes found resting in the different net types, nor in nets in different age categories (Table 3).

\section{Susceptibility of mosquito samples to insecticides}

Within Bungoma, mosquitoes reared from females collected inside nets had lower susceptibility ( $5 \%$ to deltamethrin and $34 \%$ to permethrin) compared to those from larval collections (43\% to deltamethrin and 53\% to permethrin). Mosquitoes from Bungoma, whether collected from inside nets or as larvae generally showed lower susceptibility compared to mosquitoes collected as larvae from Gem (75\% to deltamethrin and 65\% to permethrin). In Gem, there were no mosquitoes collected from inside nets (Figure 1). Based on the current WHO insecticide susceptibility guidelines, populations from Bungoma and Gem would be classified as resistant to the two insecticides [32].

\section{Mosquito bioassays}

Bed net bioassays were conducted on a sample of the field-collected nets with mosquitoes present inside $(\mathrm{N}=68)$ and compared with those in which no mosquitoes were found $(\mathrm{N}=32)$. Average knockdown and mortality were above $90 \%$ for nets that had mosquitoes present inside and for those in which no mosquitoes were observed resting inside on the day of collection (Table 4). No significant differences were observed in the knockdown or mortality of the susceptible strain to the two groups of nets (Table 4). When exposed to brand new, unused, unwashed nets, An. gambiae s.s. from Bungoma had $57.5 \%$ knockdown and $37.5 \%$ mortality to Olyset $(\mathrm{N}=40)$ and $54 \%$ knockdown and $22 \%$ mortality $(\mathrm{N}=50)$ to PermaNet 2.0 nets.

Table 2 Mean number of mosquitoes found in nets in Bungoma by the hole area

\begin{tabular}{|c|c|c|c|c|c|}
\hline $\begin{array}{l}\text { Hole index } \\
\text { category }\end{array}$ & $\begin{array}{l}\text { Sample } \\
\text { size }\end{array}$ & $\begin{array}{c}\text { Mean number of Anopheles } \\
\text { per net }\end{array}$ & $\begin{array}{l}\text { Lower } 95 \% \text { Confidence } \\
\text { intervals }\end{array}$ & $\begin{array}{l}\text { Upper } 95 \% \text { Confidence } \\
\text { intervals }\end{array}$ & $\begin{array}{c}\text { Nets with Mosquitoes } \\
\text { inside }\end{array}$ \\
\hline $0 \mathrm{~cm}^{2}$ & 163 & 1.01 & 0.67 & 1.34 & $37 \%$ \\
\hline$<50 \mathrm{~cm}^{2}$ & 55 & 1.40 & 0.73 & 2.07 & $24 \%$ \\
\hline $50-500 \mathrm{~cm}^{2}$ & 103 & 2.06 & 1.3 & 2.78 & $41 \%$ \\
\hline$>500 \mathrm{~cm}^{2}$ & 53 & 1.91 & 0.94 & 2.87 & $40 \%$ \\
\hline
\end{tabular}


Table 3 Results of a Poisson regression model of the number of An. gambiae s.l. inside ITNs in Bungoma County including the model estimates, risk ratios and P-values

\begin{tabular}{lll}
\hline Parameter & Risk Ratio & P-value \\
\hline Net type & $0.85(0.50,1.46)$ & 0.563 \\
Olyset & $0.74(0.38-1.47)$ & 0.394 \\
PermaNet & Ref. & Ref. \\
SupaNet & & \\
Net age* & $1.02(0.56-1.84)$ & 0.945 \\
Less than 3 years & Ref. & Ref. \\
Older than 3 years & & \\
Hole index category & $1.89(1.148-3.111)$ & $\mathbf{0 . 0 1 2}$ \\
$>500 \mathrm{~cm}^{2}$ & $2.08(1.38-3.12)$ & $<\mathbf{0 . 0 0 1}$ \\
50 to $500 \mathrm{~cm}^{2}$ & $1.34(0.8-2.36)$ & 0.243 \\
$<50 \mathrm{~cm}^{2}$ & Ref. & Ref. \\
No holes & &
\end{tabular}

The $95 \% \mathrm{Cl}$ for the estimate and risk ratios are given in parentheses. Significant $P$-values are given in boldface.

*Nets of unknown age were categorized as older than three years. Additional models were run with nets of unknown aged categorized as less than or equal to three years and the results did not change substantially.

\section{Molecular assays}

All the mosquitoes collected inside nets were identified as An gambiae s.s. $(\mathrm{N}=283)$, while 40 samples did not amplify and were not identified. Among mosquitoes collected by pyrethrum spray collections (PSC), 73\% were An. gambiae s.s. and $27 \%$ were $A n$. arabiensis $(\mathrm{N}=88)$ while 12 samples did not amplify. All the An. gambiae s.s. samples collected from inside the nets were homozygous for $k d r$ 1014S $(\mathrm{N}=343)$ and $1.8 \%$ of the An. gambiae s.s. collected inside nets tested positive for Plasmodium falciparum sporozoites $(\mathrm{N}=343)$. A total of $95.8 \%$ of the samples collected from PSCs in Gem were An. arabiensis with only $4.2 \%$ being $A n$. gambiae s.s. $(\mathrm{N}=48)$.

\section{Discussion}

In Bungoma, an area with high levels of pyrethroid resistance [30,36], live Anopheles mosquitoes were routinely observed resting inside nets. This may have been due to declining bioefficacy of the nets, reduced susceptibility of the mosquitoes to pyrethroid insecticides or both. Susceptibility testing using females reared from field-collected larvae or from the $\mathrm{f} 1$ generation derived from mosquitoes collected inside nets confirmed high resistance to pyrethroids. Average knockdown and mortality of a susceptible strain of mosquitoes exposed to field-collected nets was greater than $90 \%$ regardless of whether the mosquitoes had been observed resting inside, indicating that the nets in the field generally had adequate levels of insecticide: the average mortality rates were well above the $80 \%$ threshold recommended by WHOPES as a criteria for a functional ITN/LLIN [31]. In contrast, the mortality of $\mathrm{f} 1$ adult mosquitoes raised from adult mosquitoes collected inside nets in Bungoma and exposed to unused, unwashed Olyset and PermaNet 2.0 nets was 37.5 and $22 \%$, respectively. Several mosquitoes collected inside the nets were positive for P. falciparum sporozoite infection.

In contrast to Bungoma, no mosquitoes were observed inside 213 nets in Gem. Although no formal analysis was done, there were several differences between the two sites. The main difference was that the Anopheles population in Gem is comprised largely of $A n$. arabiensis. This species has lower resistance to pyrethroid insecticides [30,36], which may have reduced the likelihood

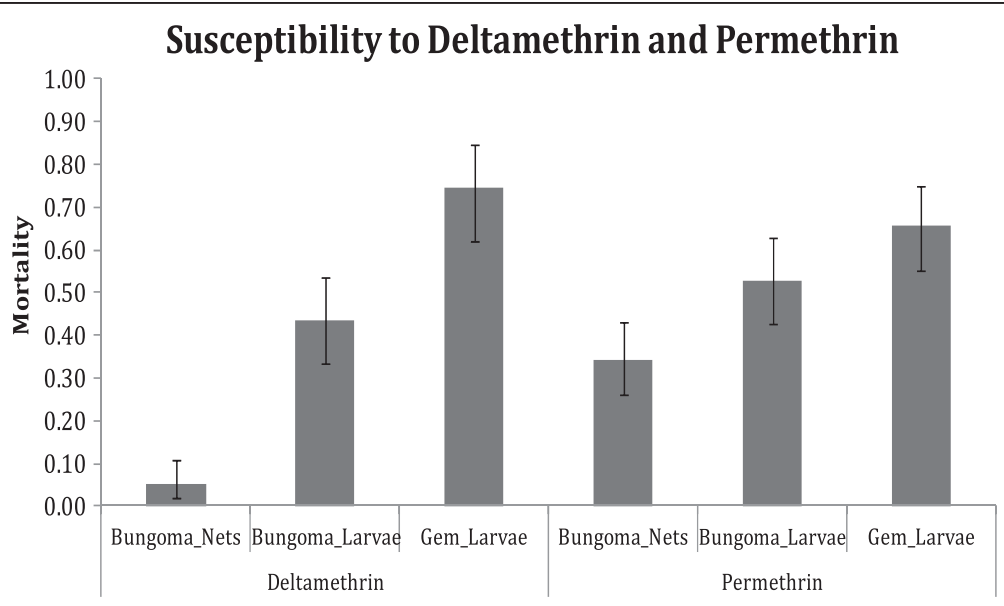

Figure 1 Susceptibility status of mosquito populations. Mortality of An. gambiae s.l. mosquito samples when exposed to deltamethrin and permethrin. Bungoma_Nets represents mortality of $\mathrm{f1}$ offspring of mosquitoes collected resting inside nets in Bungoma, Bungoma_larvae represents mortality of samples collected as larvae in Bungoma and reared to adults for exposure while Gem_larvae mortality represents mortality of samples collected as larvae in Gem and reared to adults for exposure. 
Table 4 Mean knockdown and mortality of susceptible Anopheles gambiae s.s. Kisumu strain when exposed to field collected nets with mosquitoes present $(\mathrm{N}=68)$ or absent $(\mathrm{N}=32$ ) at the time of collection and the comparison of knockdown and mortality by logistic regression

\begin{tabular}{lllll}
\hline Outcome & Mosquitoes & Mean & Odds Ratio & P-value \\
\hline Knockdown & Present & 94.4 & $1.20(0.88,1.80)$ & 0.201 \\
& Absent & 92.9 & Ref. & \\
\multirow{2}{*}{ Mortality } & Present & 92.5 & $0.74(0.51,1.06)$ & 0.108 \\
& Absent & 94.2 & Ref. & \\
\hline
\end{tabular}

The $95 \% \mathrm{Cl}$ for the estimate is given in parentheses.

that mosquitoes would enter an ITN and survive exposure. This species is also more likely to feed on alternative hosts compared to An. gambiae s.s., which may allow it to persist in presence of high coverage of ITNs [24]. Anopheles gambiae s.s. historically was very common in Gem and surrounding areas and it is still present at low levels. It has been shown to be resistant to pyrethroid insecticides (unpublished data) and it is not clear why its numbers have not rebounded or why none were observed resting inside nets. It is possible that the resistance mechanism or intensity of resistance in An. gambiae in Gem is different from that of Bungoma, where mass LLIN campaigns were first conducted in 2011. Alternatively, there may be additional ecological constraints, which in combination with the widespread use of ITNs, result in the continued suppression of the population of An. gambiae s.s. in Gem.

Pyrethroid resistance has been spreading rapidly in sub-Saharan Africa and has been documented in 23 countries [28]. This may partly be in response to agricultural application and run-off of insecticides into mosquito breeding sites [38-40], but increasingly in response to selection pressure resulting from the scale up of insecticide-treated nets and indoor residual spraying as malaria prevention tools [4,36,41-45]. Regardless of the source of insecticide pressure, insecticide resistance in malaria vectors has been predicted to eventually undermine control programmes that are solely reliant on insecticides such as indoor residual spraying (IRS) and ITN programmes [28]. While pyrethroid resistance has been documented in malaria vectors throughout subSaharan Africa, there is surprisingly little information on the impact of resistance on the effectiveness of vector control efforts. An experimental, hut trial in two sites in Benin, one with susceptible mosquitoes and the other with resistance to pyrethroids, showed blood-feeding was reduced by $96 \%$ at the site with susceptible vector population, but was largely unaffected at the site with high levels of pyrethroid resistance, while the mortality of mosquitoes entering huts at the susceptible site was nearly three times as high as that at the site with high levels of pyrethroid resistance [46]. Household trials in other parts of Benin also showed that sleeping under an ITN in an area with resistant mosquitoes was no more protective than sleeping under an untreated net, regardless of its physical condition [47]. During a longitudinal study of inhabitants of Dielmo village, Senegal, a rise in the incidence of malaria following the distribution of LLINs was attributed to increasing pyrethroid resistance in the local vector populations [48]. In contrast, a study in Ivory Coast found no reduction in the protective efficacy of ITNs in an area with high levels of pyrethroid resistance [41], while in Malawi, increasing pyrethroid resistance in An. funestus was not associated with an increase in malaria transmission in areas with LLINs although in areas with IRS, no additional impact was observed [49]. In Benin, mosquitoes were collected from inside nets with 12 holes that were $4 \mathrm{~cm} \times 4 \mathrm{~cm}$. Insecticide treated nets reduced the number of mosquitoes entering compared to an untreated net but an average of 5 mosquitoes were collected each night under LLINs [50]. A modeling study to measure the effect of pyrethroid resistance on the cost effectiveness of LLINs showed strong, positive correlations between insecticide susceptibility status and predicted population level insecticidal effectiveness of and protection against blood feeding by LLIN intervention programmes [51]. With the most resistant mosquito population, LLIN mass distributions would avert up to $40 \%$ fewer episodes of malaria compared to areas with a fully susceptible population [51]. An ongoing study in western Kenya shows prospects of generating evidence within the next year or two on the impact of insecticide resistance on the efficiency of malaria control interventions (Mbogo, pers. comm).

Several factors associated with the number of mosquitoes inside nets were explored. As described above, the location was a strong determinant of the presence of mosquitoes inside nets, presumably due to the composition of the local vector population, and further analyses included only Bungoma. In that site, neither net brand nor the age of the nets was associated with the number of mosquitoes inside nets. Although the nets were not stratified by age, the study demonstrated high mortalities of susceptible mosquitoes exposed to nets collected from the field indicating that most nets had adequate levels of insecticide. An increase in the number of mosquitoes inside nets with increasing levels of physical damage was however, observed. Nets with estimated hole areas of $>50 \mathrm{~cm}^{2}$ had more mosquitoes than nets with no holes. Although the sample sizes were limited, the data suggested that a threshold is reached beyond which increasing damage does not lead to increasing numbers of mosquitoes. This may indicate that beyond a certain amount of damage, nets are equally likely to be penetrated by mosquitoes. However, the possibility that increasing damage also allows for more mosquitoes to escape from nets, which may 
also account for the apparent threshold effect, cannot be ruled out. Interestingly, nets with no holes had an average of just over one mosquito per net. Presumably, this was due to improper usage and residents should be instructed on how to tuck their nets in to prevent mosquitoes from entering them.

It has been suggested that the physical integrity of the LLINs may be compromised before the insecticidal activity falls below established thresholds indicating the need for replacement $[49,50]$ and multiple reports have documented physical damage to nets under conditions of routine use. Rehman et al. noted that 39, 24 and 63\% of all the nets in use in Bioko Island, continental Equatorial Guinea and Malawi, respectively, were holed within two years of distribution [52]. During a long-term assessment of a polyester-based LLIN in Uganda, more than $70 \%$ of nets had holes after only one year and more than $85 \%$ after two years [53]. Wills et al., reported $54.5 \%$ of nets having holes after just six months of distribution in Ethiopia [54]. In Kenya, in an ongoing net durability study in western Kenya, it was observed that up to $40 \%$ of some net types had holes within six months of deployment in Siaya County (Bayoh, pers. comm.) while some recent surveys reported that up to $74 \%$ of the bed nets in use in Kwale County had holes [55,56]. The WHOPES guidelines on monitoring the durability of LLINs outline methods to estimate the hole sizes on the net fabric [31]. However, the guidelines do not provide criteria for physical damage that is indicative of net failure and requiring the replacement of the nets. Mutuku et al. proposed a pHI of 88 corresponding to approximately $500 \mathrm{~cm}^{2}$ of damage [55] while Gnanguenon et al. observed mosquitoes entering nets with 12 holes $4 \mathrm{~cm} \times$ $4 \mathrm{~cm}$ corresponding to a proportionate hole indexes (pHI) of 276 [50,55]. Several authors have suggested criteria based upon $\mathrm{pHI}$ and the probability that owners will discontinue use due to the owners' perception that the nets are no longer effective $[57,58]$. The cut-off for an unacceptable net ranged from a pHI of 300 in Chad corresponding to a hole area of approximately $1,000 \mathrm{~cm}^{2}$ to a pHI of 764 in Ethiopia which corresponded to a hole area of approximately $1,200 \mathrm{~cm}^{2}$. In studies in Bioko Island and Malawi, the risk of malaria increased with deteriorating condition of nets with untreated nets with at least one hole providing the least protection [52] although specific thresholds for net replacement were not presented. The data suggest that in areas with high levels of pyrethroid resistance, the threshold for a net requiring replacement may be at the lower end of the spectrum. While there is complex relationship between hole area and insecticidal activity of the nets and insecticide resistance and behaviour of the vector population, specific criteria for the physical integrity of nets should be developed to assist national malaria control programmes in determining the appropriate replacement strategies for LLINs.

The spread of pyrethroid resistance combined with increasing evidence that it may compromise malaria vector control programmes highlights the need for new insecticides and new tools for malaria prevention. Currently, LLINs are treated with pyrethroid insecticides only and their loss as an effective tool would seriously undermine malaria control programmes throughout sub-Saharan Africa. IRS with non-pyrethroid insecticides is an option that is immediately available. However, IRS is expensive relative to LLINs, particularly when spraying is done with non-pyrethroids, and is unlikely to be widely implemented without a significant increase in the amount of funding available for malaria control programmes. Two new LLIN products are currently available that incorporate a synergist to mitigate the effects of pyrethroid resistance. The Permanet 3.0 is treated with deltamethrin on the sides and deltamethrin plus piperonyl butoxide (PBO) on top. PBO is a synergist and increases the potency of the pyrethroid insecticides by inhibiting oxidase enzymes that have been implicated as one mechanism of resistance [59]. Evidence that the PermaNet 3.0 is more effective than the PermaNet 2.0 which is treated with deltamethrin alone, however, is limited and occasionally mixed, presumably due to the presence of other resistance mechanisms that are unaffected by $\mathrm{PBO}$ and the WHO Pesticide Evaluation Scheme did not recommend this product for use as a resistance management tool [60]. The Olyset Plus is another bi-treated net with permethrin plus PBO throughout the net [33]. However, there is limited data on the efficacy of this net against wild populations of mosquitoes that are resistant to pyrethroid insecticides. Larviciding is an alternative vector control tool with a wide range of activity that are recommended for use against malaria vectors. However, as with IRS, larviciding can be expensive and is currently only recommended for specific settings. Other insecticides such as chlorfenapyr, indoxacarb [46], and diafenthiuron [61] are being investigated as options for IRS but it is likely to be several years before commercially available formulations will be available. Spatial repellents [62] and toxic sugar baits [63] have also been proposed for malaria prevention but these too require several years of evaluation and refinement before they can be considered viable tools for malaria control programmes.

While this study has demonstrated that pyrethroid resistant mosquitoes are entering and surviving exposure to LLINs, the results should not be interpreted to indicate that LLINs are no longer useful in malaria control programmes. First, while pyrethroid resistance is widespread, the intensity of resistance in many areas is likely low and in these areas LLINs may still be effective. The lack of mosquitoes collected inside nets in Gem 
demonstrates that LLINs are not compromised everywhere and differences in the intensity of resistance, as well as the effectiveness of LLINs, may vary over relatively short distances. Second, this study was a crosssectional survey and differences in the age of mosquitoes may affect their susceptibility to pyrethroid insecticides as older mosquitoes have been shown to be more susceptible. Additionally, mosquitoes may repeatedly encounter insecticides over their life and, although this has not been demonstrated, the cumulative exposure may eventually result in the death of the mosquitoes. However, the finding of $P$. falciparum-infected mosquitoes inside nets suggests that, at least in Bungoma, older mosquitoes are able to survive exposure to treated nets. Lastly, intact untreated nets still provide some protection and there may be community-wide effects where malaria transmission is reduced when most people in the population regularly sleep under nets. Without baseline data on the effectiveness of LLINs before the rise of pyrethroid resistance, the impact of resistance on the effectiveness of LLINs cannot be reliably measured. However, the data strongly suggest that the efficacy of pyrethroidtreated nets may be compromised in areas with high levels of pyrethroid resistance.

\section{Conclusion}

The study found An. gambiae mosquitoes resting inside pyrethroid-treated nets in an area of documented pyrethroid resistance in western Kenya. It was confirmed that the local mosquitoes were resistant to permethrin and deltamethrin, that they survived exposure to unused, unwashed LLINs in standard cone bioassays and that fieldcollected nets retained adequate levels of insecticide as measured in bioassays against a susceptible strain of $A n$. gambiae mosquitoes. The data presented here indicate that resistance in Anopheles mosquitoes in Bungoma may be undermining the efficacy of the nets in the area. It was demonstrated that nets with holes were more likely to harbour resting mosquitoes. Given the spread of pyrethroid resistance throughout sub-Saharan Africa, it is hoped that other researchers will adopt this methods to examine how well bed nets are preventing mosquito entry in other sites. These findings highlight the need for new insecticides and tools for malaria prevention in Africa as well as the need to refine LLIN replacement strategies. Failure to address the spread of pyrethroid resistance threatens to undermine the gains made in the prevention and control of malaria in sub-Saharan Africa.

\section{Competing interests}

The authors declare that they have no competing interests.

\section{Authors' contributions}

$E O, J E G, N M B, M O, B A, C O, A K, J V, Y G$ and EDW designed the study, developed the study and took part in the manuscript preparation. EO, JEG and $\mathrm{NMB}$ contributed to development of the protocol and data analysis.
BA and EO performed the laboratory analysis of the samples. All authors read and approved the final manuscript.

\section{Acknowledgements}

We are grateful to the team of community interviewers and mosquito samplers in Bungoma and Gem, the dedicated KEMRI/CDC entomology team, specifically Celestine Wekesa, Judith Wandera, Brigid Kemei, Joseph Nduati, Diana Omoke, Duncan Ayata and Dan Juma and the KEMRI Climate and Human Health research team: Silas Agumba, Enock Onyango, Paul Omondi, Joseph Maritim, Amos Ouko, Maxwel Gesuge and Amos Wabwale for their support and dedication during the fieldwork and lab analysis of the collected samples. We thank the director KEMRI for the permission to publish this data. This study was supported by resources from the National Science Foundation (NSF grant EF-072377) and the Forgatory grant, USA, the National Council for Science and Technology (NCST), Division of Malaria Control (DOMC) and Population Services International (PSI), Kenya.

\section{Disclaimer}

The findings and conclusions in this report are those of the authors and do not necessarily represent the views of the Centers for Disease Control and Prevention.

\section{Author details}

${ }^{1}$ KEMRI/CDC Research and Public Health Collaboration, PO Box 1578, Kisumu 40100, Kenya. ${ }^{2}$ Department of Biomedical Science and Technology, School of Public Health and Community Development, Maseno University, Maseno, Kenya. ${ }^{3}$ Department of Microbiology and molecular genetics, Michigan State University, East Lansing, MI, USA. ${ }^{4}$ Centre for Global Health Research, Kenya Medical Research Institute, Kisumu, Kenya. ${ }^{5}$ Department of Ecology \& Evolutionary Biology, School of Biological Sciences, University of California, Irvine, CA, USA. ${ }^{6}$ Division of Parasitic Diseases and Malaria, Centers for Disease Control and Prevention, Atlanta, GA, USA.

Received: 23 August 2013 Accepted: 23 October 2013

Published: 24 October 2013

\section{References}

1. Alonso PL, Lindsay SW, Armstrong JRM: The effect of insecticide-treated bed nets on mortality of Gambian children. Lancet 1991, 337:1499-1502.

2. Phillips-Howard PA, Nahlen BL, Kolczak MS, Hightower AW, Kuile FO, Alaii JA, Gimnig JE, Arudo J, Vulule JM, Odhacha A, Kachur SP, Schoute E, Rosen DH, Sexton JD, Oloo AJ, Hawley WA: Efficacy of permethrin-treated bed nets in the prevention of mortality in young children in an area of high perennial malaria transmission in western Kenya. Am J Trop Med Hyg 2003, 68:23-29.

3. Lengeler $C$ : Insecticide-treated bed nets and curtains for preventing malaria. In Cochrane Database of Systematic Reviews. Chichester, UK: John Wiley \& Sons, Ltd; 2004:1-12.

4. WHO: World malaria report. Geneva: World Health Organization and UNICEF; 2005.

5. WHO: World malaria report. Geneva: World Health Organization; 2012.

6. Baume CA, Marin MC: Gains in awareness, ownership and use of insecticide-treated nets in Nigeria, Senegal, Uganda and Zambia. Malar J 2008, 7:153.

7. Macintyre K, Littrell M, Keating J, Hamainza B, Miller J, Eisele TP: Determinants of hanging and use of ITNs in the context of near universal coverage in Zambia. Health Policy Plan 2011. 10.1093/heapol/ CZr1042.

8. DOMC: 2010 Kenya MALARIA indicator survey. Nairobi, Kenya: Ministry of Public Health and Sanitation; 2011:21-22. 47-48.

9. Okiro EA, Alegana VA, Noor AM, Mutheu JJ, Juma E, Snow RW: Malaria paediatric hospitalization between 1999 and 2008 across Kenya. BMC Med 2009, 7:75.

10. Okiro E, Alegana V, Noor A, Snow R: Changing malaria intervention coverage, transmission and hospitalization in Kenya. Malar J 2010, 9:285.

11. Curtis CF, Lines JD, Carnevale P, Robert V, CB C: Impregnated nets and curtains against malaria mosquitoes. In Appropriate Technology in Vector Control. Edited by Curtis CF. Boca Raton, Florida, USA: CRC Press; 1990:5-46.

12. Irish S, N'Guessan R, Boko P, Metonnou C, Odjo A, Akogbeto M, Rowland M: Loss of protection with insecticide-treated nets against pyrethroid- 
resistant Culex quinquefasciatus mosquitoes once nets become holed: an experimental hut study. Parasit Vectors 2008, 1:17.

13. Miller JE, Lindsay SW, Armstrong JRM: Experimental hut trials of bednets impregnated with synthetic pyrethroid and organophosphate insecticides for Mosquito control in The Gambia. Med Vet Entomol 1991, 5:465-476.

14. Binka F, Indome F, Smith T: Impact of spatial distribution of permethrinimpregnated bed nets on child mortality in rural northern Ghana. Am J Trop Med Hyg 1998, 59:80-85.

15. Hawley WA, Phillips-Howard PA, TerKuile FO, Terlouw DJ, Vulule JM, Ombok M, Nahlen BL, Gimnig JE, Kariuki SK, Kolczak MS, Hightower AW: Community-wide effects of permethrin-treated bed nets on child mortality and malaria morbidity in western Kenya. Am J Trop Med Hyg 2003, 68:121-127.

16. Takken W: Do insecticide treated bednets have an effect on malaria vectors? Trop Med Int Health 2002, 7:1022-1030.

17. Choi HW, Breman JG, Teutsch SM, Liu S, Hightower AW, Sexton JD: The effectiveness of insecticide-impregnated bed nets in reducing cases of malaria infection: a meta-analysis of published results. Am J Trop Med Hyg 1995, 52:377-382.

18. Beier JC, Oster CN, Onyango FK: Plasmodium falciparum incidence relative to entomological inoculation rates at a site proposed for testing malaria vaccines in Western Kenya. Am J Trop Med Hyg 1994, 50:529-536.

19. Beier JC, Perkins PV, Onyango FK: Characterization of malaria transmission by Anopheles (Diptera: Culicidae) in western Kenya in preparation for malaria vaccine trials. J Med Entomol 1990, 27:570-577.

20. O'Meara WP, Mangeni JN, Steketee R, Greenwood B: Changes in the burden of malaria in sub-Saharan Africa. Lancet Infect Dis 2010, 10:545-555.

21. Adazu K, Hamel M, Feiken D: Marked decline in childhood mortality in the Western Kenya DSS: evidence from longitudinal data 2003-2007. New Orleans, Louisiana, USA: American Society of Tropical Medicine and Hygiene, 57th Annual Meeting; Dec 7-11; 2008. Abstract \#372.

22. Gimnig J, Kolczak MS, Hightower AW, Vulule JM, Schoute E, Kamau L, Phillips-Howard PA, Kuile FO, Nahlen BL, Hawley WA: Effect of permethrintreated bed nets on the spatial distribution of malaria vectors in western Kenya. Am J Trop Med Hyg 2003, 68:115-120.

23. Lindblade KA, Gimnig JE, Kamau L, Hawley WA, Odhiambo F, Olang G, Kuile FOT, Vulule JM, Slutsker L: Impact of sustained use of insecticide-treated bednets on malaria vector species distribution and culicine mosquitoes. J Med Entomol 2006, 43:428-432.

24. Bayoh MN, Mathias DK, Odiere MR, Mutuku FM, Kamau L, Gimnig JE, Vulule JM, Hawley WA, Hamel MJ, Walker ED: Anopheles gambiae: historical population decline associated with regional distribution of insecticidetreated bed nets in western Nyanza Province, Kenya. Malar J 2010, 9:62.

25. Zhou G, Afrane YA, Vardo-Zalik AM, Atieli H, Zhong D, Wamae P, Himeidan YE, Minakawa N, Githeko AK, Yan G: Changing patterns of malaria epidemiology between 2002 and 2010 in Western Kenya: the fall and rise of malaria. PLOS ONE 2011, 6:e20318.

26. Hamel MJ, Adazu K, Obor D, Sewe M, Vulule J, Williamson JM, Slutsker L, Feikin DR, Laserson KF: A Reversal in reductions of child mortality in Western Kenya, 2003-2009. Am J Trop Med Hyg 2011, 85:597-605.

27. Watsierah CA, Onyango RO, Ombaka JH, Abong'o BO, Ouma C: Provider knowledge of treatment policy and dosing regimen with artemetherlumefantrine and quinine in malaria-endemic areas of western Kenya. Malar J 2012, 11:436.

28. Ranson H, N'Guessan R, Lines J, Moiroux N, Nkuni Z, Corbel V: Pyrethroid resistance in African Anopheline mosquitoes: what are the implications for malaria control? Trends Parasitol 2011, 27:91-98.

29. Adazu K, Lindblade KA, Rosen DH, Odhiambo F, Ofware P, Kwach J, Eijk AMV, Decock KM, Amornkul P, Karanja D, Vulule JM, Slutsker L: Health and demographic surveillance in rural western Kenya: a platform for evaluating interventions to reduce morbidity and mortality from infectious diseases. Am J Trop Med Hyg 2005, 73:1151-1158.

30. Ochomo E, Bayoh MN, Brogdon WG, Brogdon JE, Gimnig JE, Ouma C, Vulule JM, Walker ED: Pyrethroid resistance in Anopheles gambiae s.s. and Anopheles arabiensis in western Kenya:phenotypic, metabolic and target site characterizations of three populations. Med Vet Entomol 2013, 27:156-164.

31. WHO: Guidelines for monitoring the durability of long-lasting insecticidalmosquito nets under operational conditions. WHO/HTM/NTD/ WHOPES. Geneva: World Health Organization; 2011.
32. WHO: Test procedures for insecticide resistance monitoring in malaria vector mosquitoes. Geneva: World Health Organization; 2013:6-17.

33. WHO: Report of the fifteenth WHOPES working group meeting. World Health Organization: Geneva; 2012.

34. Scott JA, Brogdon WG, Collins FH: Identification of single specimens of the Anopheles gambiae complex by polymerase chain reaction. Am J Trop Med Hyg 1993, 49:520-529.

35. Bass C, Nikou D, Donnelly MJ, Williamson MS, Ranson H, Ball A, Vontas J, Field LM: Detection of knockdown resistance $(k d r)$ mutations in Anopheles gambiae: a comparison of two new high-throughput assays with existing methods. Malar J 2007, 6:111.

36. Mathias D, Ochomo E, Atieli F, Ombok M, Bayoh N, Olang G, Muhia D, Kamau L, Vulule JM, Hamel MJ, Walker ED, Gimnig JE: Spatial and temporal variation in the kdr allele L1014S in Anopheles gambiae s.s. and phenotypic variability in susceptibility to insecticides in Western Kenya. Malar J 2011, 10:10.

37. Wirtz RA, Zavala F, Charoenvit Y, Campbell GH, Burkot TR, Schneider I, Esser KM, Beaudoin RL, Andre RG: Comparative testing of monoclonal antibodies against Plasmodium falciparum sporozoites for ELISA development. Bull World Health Organ 1987, 65:39-45.

38. Chandre F, Darriet F, Manga L, Akogbeto M, Faye O, Mouchet J, Guillet P: Status of pyrethroid resistance in Anopheles gambiae sensu lato. Bull World Health Organ 1999, 77:230-234.

39. Brooke BD, Hunt RH, Koekemoer LL, Doussou-Yovo J, Coetzee M: Evaluation of PCR assay for detection of pyrethroid insecticide resistance in the malaria vector species of the Anopheles gambiae complex (Diptera: Culicidae). J Am Mosqu Control Assoc 1999, 15:565-568.

40. Diabate A, Baldet T, Chandre F, Akogbeto M, Guiguemde TR, Darriet F, Brengues C, Guillet P, Hemingway J, Small GH, Hougard JM: The role of agricultural use ofinsecticides in resistance to pyrethroids in Anopheles gambiae s.l in Burkina Faso. Am J Trop Med Hyg 2002, 67:617-622.

41. Henry MC, Assi SB, Rogier C, Dossou-Yovo J, Chandre F, Guillet P, Carnevale P: Protective efficacy of lambda-cyhalothrin treated nets in Anopheles gambiae pyrethroid resistance areas of Cote d'Ivoire. Am J Trop Med Hyg 2005, 73:859-864.

42. Hargreaves K, Koekemoer LL, Brooke BD, Hunt RH, Mthembu J, Coetzee M: Anopheles funestus resistant to pyrethroid insecticides in South Africa. Med Vet Entomol 2000, 14:181-189.

43. Vulule J, Beach R, Atieli F, Roberts J, Mount D, Mwangi R: Reduced susceptibility of Anopheles gambiae to permethrin associated with the use of permethrin-impregnated bednets and curtains in Kenya. Med Vet Entomol 1994, 8:71-75.

44. Czeher C, Labbo R, Arzika I, Duchemin J: Evidence of increasing leu-phe knockdown resistance mutation in Anopheles gambiae from Niger following a nationwide long-lasting insecticide-treated nets implementation. Malar J 2008, 7:189.

45. WHO: Malaria vector control and personal protection, WHO Technical Report series 936. Geneva: World Health Organization; 2006.

46. N'Guessan R, Corbel V, Akogbeto M, Rowland M: Reduced efficacy of insecticide-treated nets and indoor residual spraying for malaria control in pyrethroid resistance area, Benin. Emerg Infect Dis 2007, 13:199-206.

47. Asidi A, N'Guessan R, Akogbeto M, Curtis C, Rowland M: Loss of household protection from use of insecticide-treated nets against pyrethroidresistant mosquitoes, benin. Emerg Infect Dis 2012, 18:1101-1106.

48. Trape JF, Tall A, Diagne N, Ndiath O, Ly AB, Faye J, Dieye-Ba F, Roucher C, Bouganali C, Badiane A, Sarr FD, Mazenot C, Touré-Baldé A, Raoult D, Druilhe P, Mercereau-Puijalon O, Rogier C, Sokhna C: Malaria morbidity and pyrethroid resistance after the introduction of insecticide-treated bednets and artemisinin-based combination therapies: a longitudinal study. Lancet Infect Dis 2011, 11:925-932.

49. Wondji CS, Coleman M, Kleinschmidt I, Mzilahowa T, Irving H, Ndula M, Rehman A, Morgan J, Barnes KG, Hemingway J: Impact of pyrethroid resistance on operational malaria control in Malawi. Proc Natl Acad SC U S A 2012, 109:19063-19070.

50. Gnanguenon V, Azondekon R, Oke-Agbo F, Sovi A, Osse R, Padonou G, Aikpon R, Akogbeto MC: Evidence of man-vector contact in torn longlasting insecticide-treated nets. BMC Public Health 2013, 13:751.

51. Briet OJ, Penny MA, Hardy D, Awolola TS, Van Bortel W, Corbel V, Dabire RK, Etang J, Koudou BG, Tungu PK, Chitnis N: Effects of pyrethroid resistance on the cost effectiveness of a mass distribution of long-lasting insecticidal nets: a modelling study. Malar J 2013, 12:77. 
52. Rehman AM, Coleman M, Schwabe C, Baltazar G, Matias A, Gomes IR, Yellott L, Aragon C, Nchama GN, Mzilahowa T, Rowland M, Kleinschmidt I: How much does malaria vector control quality matter: the epidemiological impact of holed nets and inadequate indoor residual spraying. PLOS ONE 2011, 6:e19205.

53. Kilian A, Byamukama W, Pigeon O, Atieli F, Duchon S, Phan C: Long-term field performance of a polyester-based long-lasting insecticidal mosquito net in rural Uganda. Malar J 2008, 7:49.

54. Wills AB, Smith SC, Anshebo GY, Graves PM, Endeshaw T, Shargie EB, Damte M, Gebre T, Mosher AW, Patterson AE, Tesema YB, Richards FO Jr, Emerson PM: Physical durability of PermaNet 2.0 long-lasting insecticidal nets over three to 32 months of use in Ethiopia. Malar J 2013, 12:242.

55. Mutuku FM, Khambira M, Bisanzio D, Mungai P, Mwanzo I, Muchiri EM, King $\mathrm{CH}$, Kitron U: Physical condition and maintenance of mosquito bed nets in Kwale County, coastal Kenya. Malar J 2013, 12:46.

56. Githinji S, Herbst S, Kistemann T, Noor AM: Mosquito nets in a rural area of western Kenya: ownership, use and quality. Malar J 2010, 9:250

57. Kilian A, Byamukama W, Pigeon O, Gimnig J, Atieli F, Koekemoer L, Protopopoff $\mathrm{N}$ : Evidence for a useful life of more than three years for a polyester-based long-lasting insecticidal mosquito net in Western Uganda. Malar J 2011, 10:299.

58. Batisso E, Habte T, Tesfaye G, Getachew D, Tekalegne A, Kilian A, Mpeka B, Lynch C: A stitch in time: a cross-sectional survey looking at long lasting insecticide-treated bed net ownership, utilization and attrition in SNNPR, Ethiopia. Malar J 2012, 11:183.

59. Kakko I, Toimela T, Tahti H: Piperonyl butoxide potentiates the synaptosome ATPase inhibiting effect of pyrethrin. Chemosphere 2000 40:301-305.

60. WHO: Report of the twelfth WHOPES working group meeting. Geneva: World Health Organization; 2009.

61. Paul A, Harrington LC, Scott JG: Evaluation of novel insecticide for control of dengue vector Aedes aegypti (Diptera:Culicidae). J Med Entomol 2006, 45:55-60.

62. Achee NL, Bangs MJ, Farlow R, Killeen GF, Lindsay S, Logan JG, Moore SJ, Rowland M, Sweeney K, Torr SJ, Zwiebel L, Grieco JP: Spatial repellents: from discovery and development to evidence-based validation. Malar J 2012, 11:164.

63. Beier JC, Muller GC, Gu W, Arheart KL, Schlein Y: Attractive toxic sugar bait (ATSB) methods decimate populations of Anopheles malaria vectors in arid environments regardless of the local availability of favoured sugarsource blossoms. Malar J 2012, 11:31.

doi:10.1186/1475-2875-12-368

Cite this article as: Ochomo et al.: The efficacy of long-lasting nets with declining physical integrity may be compromised in areas with high levels of pyrethroid resistance. Malaria Journal 2013 12:368.

\section{Submit your next manuscript to BioMed Central and take full advantage of:}

- Convenient online submission

- Thorough peer review

- No space constraints or color figure charges

- Immediate publication on acceptance

- Inclusion in PubMed, CAS, Scopus and Google Scholar

- Research which is freely available for redistribution

Submit your manuscript at www.biomedcentral.com/submit
( Biomed Central 